ARTICLE

DOI: $10.1038 / s 41467-018-05888-w$

\title{
Single photon emission from graphene quantum dots at room temperature
}

Shen Zhao (1) ${ }^{1}$, Julien Lavie ${ }^{2}$, Loïc Rondin (1) 1, Lucile Orcin-Chaix ${ }^{1,2}$, Carole Diederichs ${ }^{3}$, Philippe Roussignol ${ }^{3}$, Yannick Chassagneux ${ }^{3}$, Christophe Voisin (10 ${ }^{3}$, Klaus Müllen ${ }^{4}$, Akimitsu Narita (1) ${ }^{4}$, Stéphane Campidelli (i) ${ }^{2}$ \& Jean-Sébastien Lauret (iD ${ }^{1}$

Graphene being a zero-gap material, considerable efforts have been made to develop semiconductors whose structure is compatible with its hexagonal lattice. Size reduction is a promising way to achieve this objective. The reduction of both dimensions of graphene leads to graphene quantum dots. Here, we report on a single-emitter study that directly addresses the intrinsic emission properties of graphene quantum dots. In particular, we show that they are efficient and stable single-photon emitters at room temperature and that their emission wavelength can be modified through the functionalization of their edges. Finally, the investigation of the intersystem crossing shows that the short triplet lifetime and the low crossing yield are in agreement with the high brightness of these quantum emitters. These results represent a step-forward in performing chemistry engineering for the design of quantum emitters.

\footnotetext{
${ }^{1}$ Laboratoire Aimé Cotton, CNRS, Univ. Paris-Sud, ENS Cachan, Université Paris-Saclay, bat 505 campus d'Orsay, 91405 Orsay cedex, France. ${ }^{2}$ LICSEN, NIMBE, CEA, CNRS, Université Paris-Saclay, Gif sur Yvette 91191, France. ${ }^{3}$ Laboratoire Pierre Aigrain, Département de physique de l'ENS, École normale supérieure, Université Paris Diderot, Sorbonne Paris Cité, Sorbonne Université, CNRS, PSL University, 75005 Paris, France. ${ }^{4}$ Max Planck Institute for Polymer Research, Ackermannweg 10, 55128 Mainz, Germany. Correspondence and requests for materials should be addressed to

J.-S.L. (email: lauret@ens-paris-saclay.fr)
} 
G raphene plays a central role as an emerging material for nanoelectronics. Nevertheless, graphene is a semimetal, which constitutes a severe limitation for some future applications. Therefore, considerable efforts are being made to develop semiconductor materials whose structure is compatible with the graphene lattice. In this perspective, little pieces of graphene are very promising ${ }^{1,2}$. In particular, their electronic, optical, and spin properties can be in principle controlled by designing their size, shape and edges ${ }^{3-6}$.

To date, graphene quantum dots (GQDs) have been mostly synthesized by top-down approaches, such as the oxidation of carbon fibers or graphene $e^{7,8}$. These methods permit to produce easily large quantities of nanoparticles but they also present important drawbacks that make them not suitable to produce emitters with well-defined properties. In particular, they do not allow to control neither the size nor the chemical nature of the edges of the dots. Therefore, their optical properties are dominated by defect states instead of the intrinsic confined states of the nanographene ${ }^{9}$. Fortunately, over the last two decades, bottom-up synthesis have been developed opening the way to a precise control of the GQD structure ${ }^{2}$. Several sizes and shapes of GQDs have already been synthesized ${ }^{1}$. However, these materials often face problems, in particular aggregation, that hinder the possibility to make the link between their structure and their intrinsic properties, especially in solid state or at the singleemitter level ${ }^{1,10}$.

Here, we report on a single-emitter study that directly addresses the intrinsic emission properties of GQDs synthesized by this bottom-up approach. We show that graphene quantum dots are efficient and stable single-photon emitters at room temperature. A control of the emission wavelength $(\sim 100 \mathrm{~nm}$ redshift) is obtained through the functionalization of the graphene quantum dot edges. Finally, the intersystem crossing (ISC) has been investigated. The short triplet lifetime and the low ISC yield agree with the high brightness of these new quantum emitters. These results represent a step-forward in performing chemistry engineering for the design of quantum emitters.

\section{Results and discussion}

Samples. The chemical structure of the two type of GQDs studied here is displayed on Fig. 1a. They are both made of $96 \mathrm{sp}^{2}$ carbon atoms arranged in a triangular shape which leads to lateral sizes of $\sim 2 \mathrm{~nm}$. Six alkyl chains $\left(\mathrm{R}=\mathrm{C}_{12} \mathrm{H}_{25}\right)$ were introduced at the edges of the first GQD to enhance its solubility. The edges of the second GQD have been functionalized with chlorine atoms to modify the optical properties. In the following, we call the two structures $\mathrm{C}_{96}$ and $\mathrm{C}_{96} \mathrm{Cl}$ GQDs, respectively. The details of the synthesis have been already reported ${ }^{3,11}$ and are described in the Supplementary Methods section. Briefly, the $\mathrm{C}_{96}$ GQD is synthesized in two steps from the 1,3,5-triethynylbenzene and 2,5-diphenyl-3,4-di(4-dodecylphenyl)-cyclopentadien-1-one via Diels-Alder cycloaddition followed by oxidative cyclodehydrogenation in the presence of $\mathrm{FeCl}_{3}$ (See Supplementary Fig. 1). The $\mathrm{C}_{96} \mathrm{Cl}$ GQD is synthesized in three steps from the 1,3,5triethynylbenzene and 2,3,4,5-tetraphenyl-cyclopentadien-1-one via Diels-Alder cycloaddition followed by the oxidation of the dendrimer via the Scholl reaction and the chlorination of the bare $\mathrm{C}_{96}$ particle by treatment with $\mathrm{ICl}$ in the presence of $\mathrm{AlCl}_{3}$ (see Supplementary Fig. 1). The intermediate compounds are fully characterized and the GQDs are characterized by MALDI-TOF spectrometry to confirm the complete dehydrogenation and chlorination (See Supplementary Fig. 2). The GQDs are dispersed in 1,2,4-trichlorobenzene and then mixed with a solution of polystyrene (PS). The mixture is subsequently spin-coated on a coverslip to perform the optical experiments, using the experimental setup described in Fig. 1b. Complementary details about the sample preparation can be found in the Methods section.

Photoluminescence spectroscopy. Figure 1c shows an example of a photoluminescence (PL) map of $\mathrm{C}_{96}$ GQDs embedded in PS matrix for the highest dilution used in this study (see also Supplementary Fig. 10). One can observe bright spots ( 25 kcounts s $^{-1}$ with $200 \mathrm{nW}$ excitation) with diffraction limited size. The PL spectrum acquired on such a spot is displayed on Fig. 1d. For this particular $\mathrm{C}_{96}$ GQD, the spectrum is composed of three lines, noted 1,2, and 3, centered at 653, 719, and $797 \mathrm{~nm}$, respectively. The full width at half maximum of the main line is of the order of $27 \mathrm{~nm}(80 \mathrm{meV})$. Note that the wavelength as well as the relative intensity of the PL lines slightly vary from one GQD to another, which is certainly due to differences in their local environments. Also, averaging over 25 GQDs gives an energy splitting of $170 \pm 5$ meV between lines 1 and 2 , and $165 \pm 10 \mathrm{meV}$ between lines 2 and 3. Moreover, as mentioned in the introduction, one of the great potential of GQDs lies in the precise tuning of their electronic properties through the control of their structure. As a first example, the dotted curve in Fig. 1d shows the PL spectrum of a single $\mathrm{C}_{96} \mathrm{Cl}$ GQD whose emission wavelength has been shifted by the chemical functionalization of the edges with chlorine atoms. Here the functionalization leads to an almost $100 \mathrm{~nm}$ rigid redshift of the main PL line. This observation is in agreement with theoretical predictions that show a decrease of the optical gap of $\mathrm{C}_{96} \mathrm{Cl}$ in comparison with $\mathrm{C}_{96}$ GQD due to the electronegativity of the chlorine atoms ${ }^{11}$. This result illustrates how the intrinsic properties of GQDs can be controlled by the synthesis.
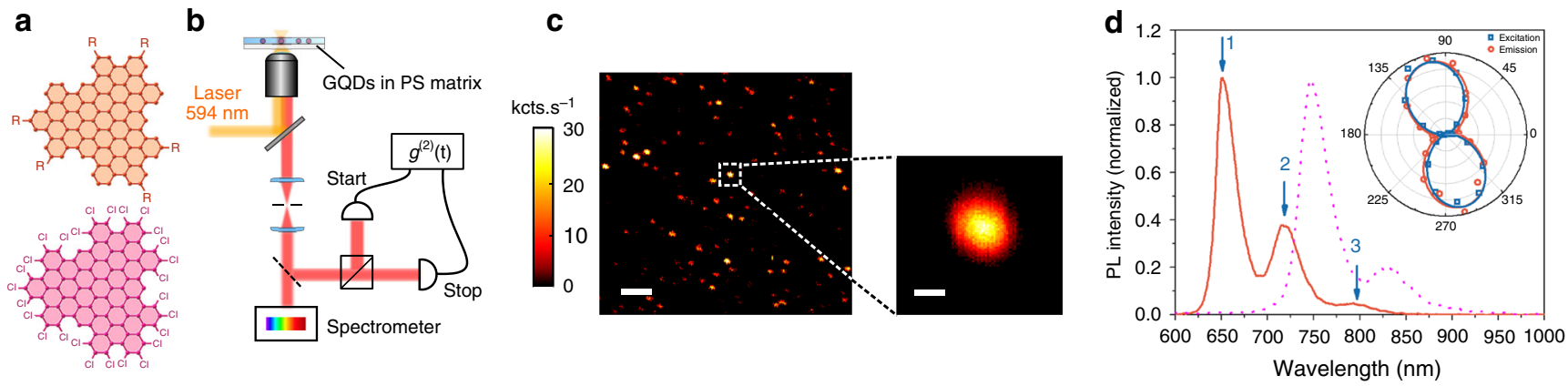

Fig. 1 Photoluminescence of single GQDs. a Chemical structure of the $\mathrm{C}_{96}$ and $\mathrm{C}_{96} \mathrm{Cl}$ GQDs. $R$ stands for $\mathrm{C}_{12} \mathrm{H}_{25}$. b Scheme of the microphotoluminescence setup. c $20 \times 20 \mu \mathrm{m}^{2} \mathrm{PL}$ map of the $\mathrm{C}_{96}$ GQDs in polystyrene matrix. The color bar represents the number of counts per second on the APD. The scale bar is $3 \mu \mathrm{m}$. The zoom shows a diffraction limited spot that can be fitted with a 2D Gaussian function leading to a 1/e diameter of $\sim 600 \mathrm{~nm}$. The scale bar is $200 \mathrm{~nm}$. d Room temperature PL spectra of a single $\mathrm{C}_{96}$ GQD (solid line) and of a single $\mathrm{C}_{96} \mathrm{Cl}$ GQD (dotted line). Polarization diagram in excitation (blue) and emission (red) 
In the following, we focus on $\mathrm{C}_{96}$ GQDs containing the $\mathrm{C}_{12} \mathrm{H}_{25}$ alkyl chains. First we investigate the nature of the quantum states at the origin of the three PL lines. The energy splitting between each line is $\sim 170 \mathrm{meV}$ very close to the $\mathrm{C}=\mathrm{C}$ stretching vibration mode. Moreover, theoretical works on this family of objects indicate the existence of low energy dark states, the lowest one being called the $\alpha$ band in the Clar's notation ${ }^{12,13}$. The coupling with the vibrations of the lattice can lead to a brightening of these low energy states ${ }^{14}$. Here, we attribute the first PL line to the zerophonon emission line of the $\alpha$ band followed up with vibronic replicas, with a quantum of vibration $\hbar \Omega \sim 170 \mathrm{meV}$. We also performed photoluminescence excitation (PLE) experiments in solution (See Supplementary Fig. 4). The PLE curves detected on the two highest energy PL lines of GQD superimpose well with the absorption spectrum of the solution. Besides, the PLE highlights the existence of two lines at 580 and $630 \mathrm{~nm}$. The energy splitting between those states is also $\sim 170 \mathrm{meV}$ revealing the Franck-Condon series of vibronic lines. The intense absorption line at high energy could be tentitatively attributed to the $\beta$ band in the Clar's notation ${ }^{12,13}$. Finally, the polarization response of a single GQD is shown in the inset of Fig. 1d. Here, the emission polarization profile is recorded on the line 1 at $\sim 650$ $\mathrm{nm}$. It is linearly polarized at a fixed direction certainly related to the geometry of the GQD. Likewise the excitation diagram is also linearly polarized in the same direction than the emission one. Therefore, it can be concluded that absorption and main emission dipoles are parallel. Further investigations, including theoretical modeling, are needed to explain this experimental observation.

Second-order correlation function. In order to identify the number of emitters associated with such diffraction limited spot and spectrum, we measured the second-order correlation function $\left(g^{(2)}(\tau)\right)$ at room temperature integrating over the entire spectrum. As displayed in Fig. 2a, the strong antibunching observed at zero delay, $g^{(2)}(0)<0.1$, is a proof that a single emitter is detected. This is in strong contrast with the results of such experiments performed on top-down GQDs where no antibunching is observed ${ }^{9}$. In these earlier studies, the absence of antibunching is interpreted as a consequence of the extrinsic nature of the states at the origin of the luminescence, leading to multidefect sites emitting in an uncorrelated manner ${ }^{9}$. We have performed measurements on more than 30 GQDs, all of them leading to $g^{(2)}(0)<0.1$ (see examples on Supplementary Fig. 5). Moreover, these correlation measurements being performed by integrating all the wavelengths on the detector, implies that the spectrum described above actually arises from a single GQDs and not from several objects. Moreover, the weak value observed for the $g^{(2)}(0)$ is an indication of the good purity of single-photon emission associated with single GQD. This result enforces GQD as an interesting alternative to other single emitters ${ }^{15}$, such as defects in $\mathrm{WSe}_{2}{ }^{16-20}$, in $h-\mathrm{BN}^{21-23}$ or in carbon nanotubes ${ }^{24}$. a

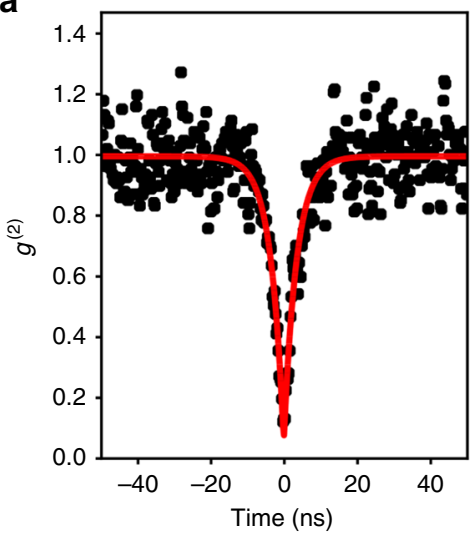

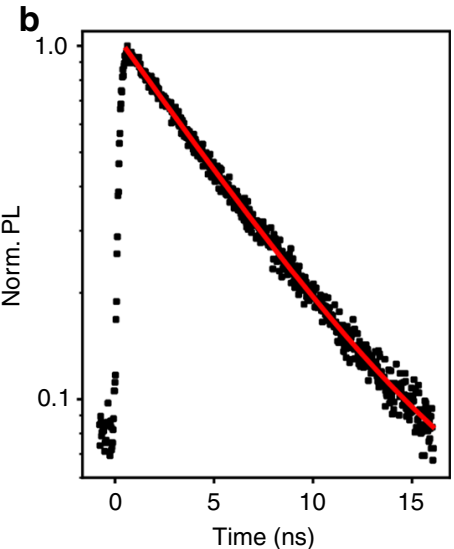

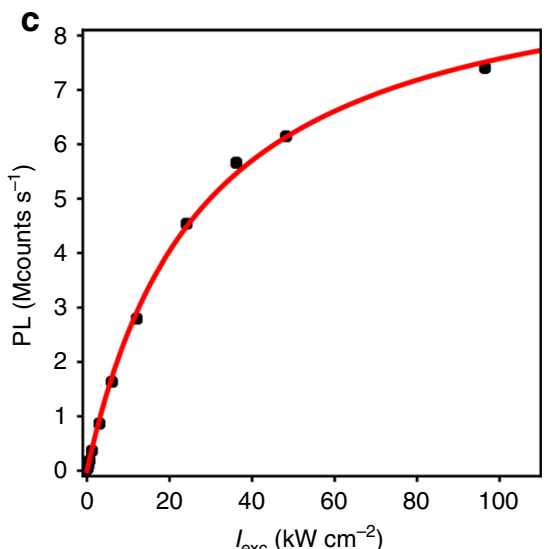

d

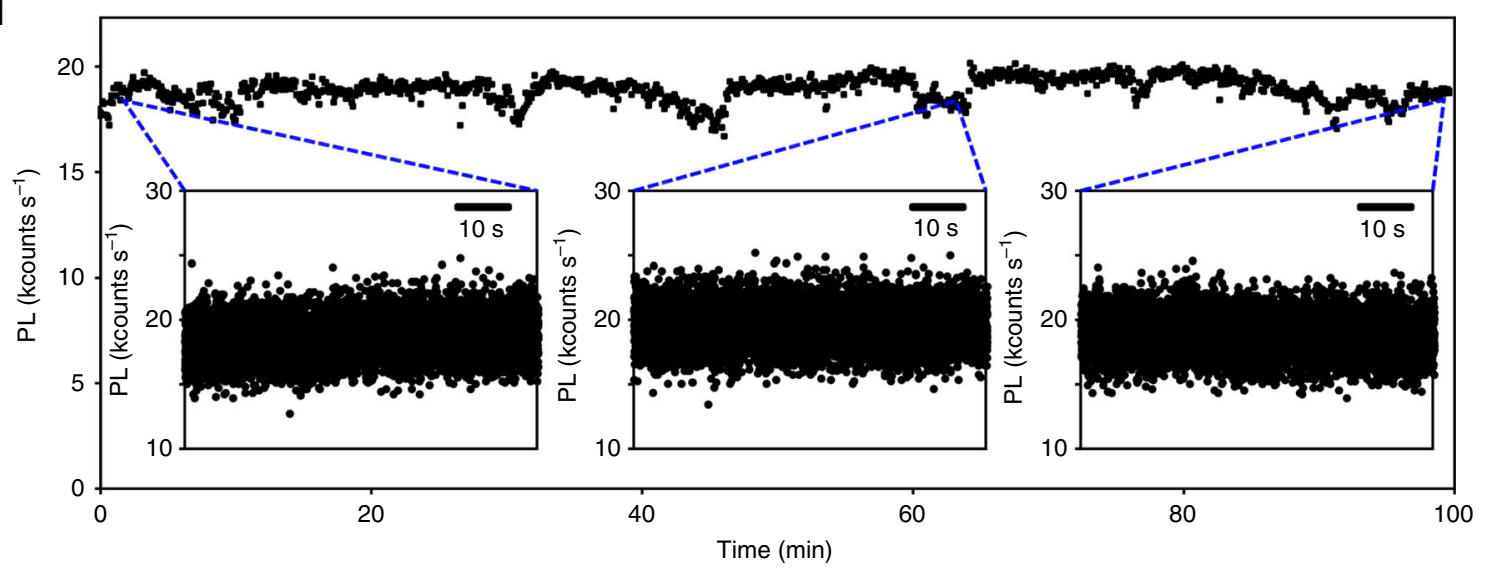

Fig. 2 Photophysics of a single GQD. a Second-order correlation function $g^{(2)}(\tau)$ recorded from a diffraction limited spot such as the one of Fig. 1 (black dots), showing a strong antibunching. A fit (red line) with a function $1-(1-b) e^{-|\tau| / \tau_{1}}$ yields $g^{(2)}(0)=0.05 \pm 0.05$ and a characteristic time of $\tau_{1} \sim 3.5 \mathrm{~ns}$ (FWHM of the IRF of the detector $\sim 0.9 \mathrm{~ns}$ ). $\mathbf{b}$ Time-resolved PL of a single GQD (black dots) detected on the whole spectrum, fitted by a monoexponential decay (red line) with a time constant $\tau=5.37 \mathrm{~ns}$. c Saturation curve of a single GQD (black dots) as a function of the pump power. A fit by Eq. (1) (red line) leads to a saturation power density of $28 \mathrm{~kW} \mathrm{~cm}^{-2}$ and a saturation intensity $I_{\text {sat }} \sim 9.7$ Mcounts/s. $\mathbf{d}$ PL time trace of a stable GQD over 100 min with a binning time of $200 \mathrm{~ms}$. Fluctuations are due to setup instabilities. Zooms are shown on shorter timescale with a binning time of $10 \mathrm{~ms}$ 
Photophysical properties. The fact that we are actually observing single objects allows us to characterize other important properties. First, another figure of merit of a single quantum emitter is its brightness. A saturation curve of a single GQD is displayed on Fig. 2c. The intensity is fitted by

$$
R=R_{\mathrm{sat}} /\left(1+\frac{I_{\mathrm{sat}}}{I_{\mathrm{exc}}}\right),
$$

with $R_{\text {sat }}$ the count rate at saturation, $I_{\text {sat }}$ the incident power density at saturation and $I_{\text {exc }}$ the incident power density. The fit leads to $R_{\text {sat }} \sim 9.7$ Mcounts $\mathrm{s}^{-1}$, and $I_{\text {sat }}=28 \mathrm{~kW} \mathrm{~cm}^{-2}$. This value of count rate at saturation can be compared to other new quantum emitters such as defects in $2 \mathrm{D}$ materials. In this context, L.J. Martínez et al. have compared on the same setup the count rate at saturation of $\mathrm{N}-\mathrm{V}$ centers and single defects in $h-\mathrm{BN}^{22}$. We decided to employ the same method in order to quantitatively compare GQDs and single defects in $h$-BN (see Supplementary Note 3 for details). It turns that GQDs are, at least, as bright as the brightest single-photon source found in $2 \mathrm{D}$ materials. Therefore, it puts GQDs in the highest values of brightness among other quantum emitters $15,22,23$. Next, we address the question of the photostability of GQDs. In this perspective, GQDs have also good properties. Indeed, photostability up to hours have been observed for an incident power of $200 \mathrm{nW}\left(0.12 \mathrm{~kW} \mathrm{~cm}^{-2}\right)$ and an emission rate of $\sim 20$ kcounts.s $^{-1}$ (see Fig. $2 \mathrm{~d}$ and Supplementary Note 5). This encouraging result could even be improved by engineering the surrounding matrix, as it has been done for small molecules 25,26 . Moreover, Fig. $2 \mathrm{~d}$ shows that no blinking is observed on the time trace of the luminescence of this GQD. The histogram of intensity fits well to a normal distribution (see Supplementary Note 4 and Supplementary Fig. 7). This observation is in strong contrast with numerous single emitters that undergo quantum jumps between bright and dark states. Nevertheless, the luminescence of GQDs end up disappearing. Supplementary Fig. 8 shows two different time traces around the time of bleaching. In the first one, the luminescence drops down to zero sharply. On the contrary, the second one goes through an intermediate gray state before ending up to zero. These two behaviors are representative of what we observed on the GQDs. The discrete intensity jumps are also characteristic of singleemitter experiments. In order to get insights in the recombination dynamics, time-resolved photoluminescence (TR-PL) experiments on single GQDs have been performed (see Fig. $2 b$ and Supplementary Fig. 9). Here, the signal can be fitted by a monoexponential decay with a time constant $\tau \sim 5.37 \mathrm{~ns}$. We have performed such experiments on several GQDs. The TR-PL signal is always mono-exponential with relaxation times ranging from 3 to $5.5 \mathrm{~ns}$.

Finally, in order to get more information on the photophysics of GQDS, we studied the $g^{(2)}$ function on a longer timescale. In particular, such measurement allows highlighting ISC dynamics between singlet and triplet states ${ }^{27}$. This approach is also supported by calculations on GQDs showing that at least one triplet state is lying few hundreds of $\mathrm{meV}$ below the singlet state ${ }^{28,29}$. The $g^{(2)}$ functions recorded under different excitation density both at short and long-time delays are shown, respectively, in Fig. 3a, b. At short delays, one observes an acceleration of the dynamics of the $g^{(2)}$ function when the pump is increased. Likewise, at longer delays photon bunching $\left(g^{(2)}>1\right)$ is observed with a relaxation down to $g^{(2)}=1$ within tens of microseconds. These data are well fitted assuming a three-level system model, as shown in the inset of Fig. $3 \mathrm{~b}$ where state 3 stands for the triplet state. It leads to the evaluation of the transition rates: $k_{21} \sim 0.28 \mathrm{~ns}^{-1}, k_{23} \sim 0.025 \mu \mathrm{s}^{-1}$, and $k_{31} \sim 0.057$ $\mu^{-1}$ (see Supplementary Note 1 for the details of the
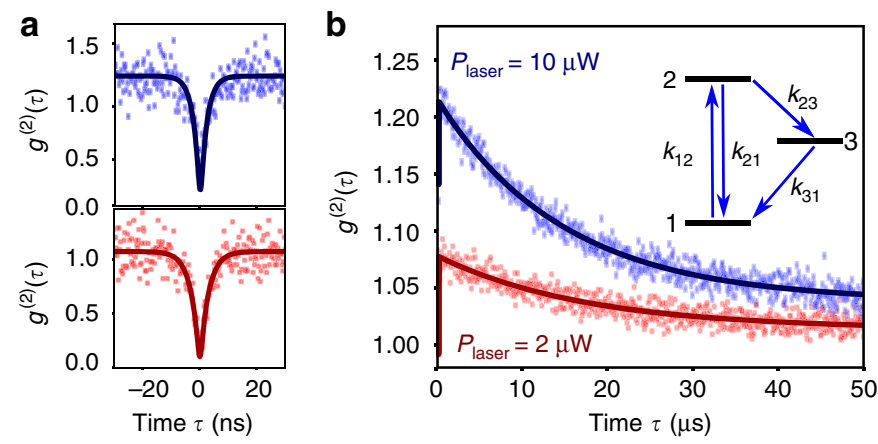

Fig. 3 Photons bunching of a single GQD. $\mathbf{a}, \mathbf{b} g^{(2)}$ functions of a single GQD, for two different excitation powers, $2 \mu \mathrm{W}$ (red square) and $10 \mu \mathrm{W}$ (blue diamond). a Zoom on short delays; b Full timescale intensity correlation. The solid line is a fit to the function $1-(1+a) e^{-\lambda_{1}|\tau|}+a e^{-\lambda_{2}|\tau|}$, convolved by the time response of the detector. The three-level system used as a model is shown

photophysics). The ISC yield $k_{23} / k_{21}$ being small $\left(\sim 10^{-4}\right)$ and the triplet lifetime being short $\left(1 / k_{31} \sim 18 \mu \mathrm{s}\right)$, the effect of the triplet on the emission efficiency is very limited. It can explain both the high brightness of GQDs and the absence of measurable blinking (see also Supplementary Fig. 12). Finally, we also provide an estimation of the absorption cross section of the $\mathrm{C}_{96} \mathrm{GQD}(\sigma$ $\left.\simeq 1.0 \times 10^{-14} \mathrm{~cm}^{2}\right)$, as well as of the PL quantum efficiency $\eta_{Q}$ above 35\% (see Supplementary Note 2).

To conclude, the good properties of GQDs: single-photon emission, brightness, and photostability show that they are very promising materials for applications requiring single emitters. More generally, the results reported here demonstrate that the high potential of GQDs revealed by theory is now accessible experimentally. In particular, the observation of single GQD emission paves the way to studies linking their properties to their structure. In addition, our results show that the modification of the properties through the control of the structure is indeed achievable. Therefore, one can now really imagine to perform engineering of other properties such as the spin structure in order to rend it optically detectable and controllable.

\section{Methods}

Techniques. MALDI-OF spectra were performed with a Perspective Biosystems Voyager DE-STR at the I.C.S.N. (CNRS of Gif-sur-Yvette). ${ }^{1} \mathrm{H}-\mathrm{NMR}$ spectra were recorded on Bruker AC-300 spectrometer $(300 \mathrm{MHz})$ with $\mathrm{CDCl}_{3}$ as reference solvent.

Materials. Chemicals were purchased from Aldrich and were used as received. The 3,4-bis(4-dodecylphenyl)-2,5-diphenylcyclopentadienone was prepared according to the method described in the literature ${ }^{30}$. Solvents were purchased from Aldrich, VWR or ThermoFisher and were used as received.

Synthesis. See Supplementary Methods.

Sample preparation. GQDs powder was dispersed in 1,2,4-trichlorobenzene (TCB) by stirring at least $24 \mathrm{~h}$ at room temperature. For single-molecule measurements, a $2 \mathrm{~mL}$ diluted GQDs solution $\left(0.001 \mathrm{mg} \mathrm{mL}^{-1}\right)$ was mixed with a $2 \mathrm{~mL}$ 1,2,4-trichlorobenzene solution $\left(0.08 \mathrm{mg} \mathrm{mL}^{-1}\right)$ of polystyrene (PS). Approximately $20 \mu \mathrm{L}$ of the GQD-PS-TCB solution was spin-coated on an oxygen-plasmatreated glass coverslip for $180 \mathrm{~s}$ at 2000 r.p.m. The sample was dried by heating to $90^{\circ} \mathrm{C}$ for $1 \mathrm{~h}$ on a hot plate. The thickness of the layer has been estimated to 25-50 $\mathrm{nm}$ by profilometry.

Optical measurements. Optical experiments were performed on a home-built micro-PL setup under ambient conditions, as shown in Fig. 1b. The excitation source was a continuous-wave diode laser at $594 \mathrm{~nm}$ (Cobolt, Mambo 100) with linear polarization. The excitation laser was focused onto the sample with a high numerical aperture oil-immersion microscope objective (NA $=1.42$, Olympus PLAPON 60XO) mounted on a piezoelectric XYZ scanner (Mad City Labs Inc.). 
The luminescence light was collected by the same objective and filtered from the residual excitation laser using a dichroic mirror (zt 594 RDC, Chroma) and a longpass filter (FELH0600, Thorlabs). The collected luminescence was then focused on a $50-\mu \mathrm{m}$-diameter pinhole and finally directed either into a spectrometer (SP-2358, Princeton Instruments) coupled with a cooled CCD camera (PyLoN:100BR eXcelon, Princeton Instruments) or into two silicon avalanche photodiodes (SPCM-AQR-13, PerkinElmer) mounted in a Hanbury Brown and Twiss (HBT) configuration. Short-time-scale second-order photon correlation measurements were done using a time-correlated single-photon counting module (PicoHarp300, PicoQuant). Long-time-scale second-order photon correlation measurements were done using a wide-range time digitizer (P7887, FastComtec). For time-resolved PL measurements, the sample was excited using a supercontinuum laser (Fianium) tuned at $580 \mathrm{~nm}$ by an acousto-optic tunable filter systems with a $6 \mathrm{ps}$ pulse width and a $60 \mathrm{MHz}$ repetition rate.

Data availability. The data that support the findings of this study are available from the corresponding author upon reasonable request.

Received: 26 June 2018 Accepted: 1 August 2018

Published online: 27 August 2018

\section{References}

1. Müllen, K. Evolution of graphene molecules: structural and functional complexity as driving forces behind nanoscience. ACS Nano 8, 6531-6541 (2014).

2. Wu, J., Pisula, W. \& Müllen, K. Graphenes as potential material for electronics. Chem. Rev. 107, 718-747 (2007).

3. Tomović, Ž., Watson, M. D. \& Müllen, K. Superphenalene-based columnar liquid crystals. Angew. Chem. Int. Ed. 43, 755-758 (2004).

4. Debije, M. G. et al. The optical and charge transport properties of discotic materials with large aromatic hydrocarbon cores. J. Am. Chem. Soc. 126, 4641-4645 (2004).

5. Yan, X., Cui, X. \& Li, L.-S. Synthesis of large, stable colloidal graphene quantum dots with tunable size. J. Am. Chem. Soc. 132, 5944-5945 (2010).

6. Konishi, A. et al. Synthesis and characterization of teranthene: a singlet biradical polycyclic aromatic hydrocarbon having kekulé structures. J. Am. Chem. Soc. 132, 11021-11023 (2010).

7. Li, L. et al. Focusing on luminescent graphene quantum dots: current status and future perspectives. Nanoscale 5, 4015-4039 (2013).

8. Bacon, M., Bradley, S. J. \& Nann, T. Graphene quantum dots. Part. Part. Sys. Charact. 31, 415-428 (2014).

9. Xu, Q. et al. Single-particle spectroscopic measurements of fluorescents graphene quantum dots. ACS Nano 7, 10654-10661 (2013).

10. Zhao, S. et al. Fluorescence from graphene nanoribbons of well-defined structure. Carbon N. Y. 119, 235-240 (2017).

11. Tan, Y.-Z. et al. Atomically precise edge chlorination of nanographenes and its application in graphene nanoribbons. Nat. Commun. 4, 2646 (2013).

12. Rieger, R. \& Müllen, K. Forever young: polycyclic aromatic hydrocarbons as model cases for structural and optical studies. J. Phys. Org. Chem. 23, 315-325 (2010).

13. Cocchi, C., Prezzi, D., Ruini, A., Caldas, M. J. \& Molinari, E. Anisotropy and size effects on the optical spectra of polycyclic aromatic hydrocarbons. J. Phys. Chem. A 118, 6507-6513 (2014).

14. Sprafke, J. K. et al. Belt-shaped П-systems: relating geometry to electronic structure in a six-porphyrin nanoring. J. Am. Chem. Soc. 133, 17262-17273 (2011).

15. Aharonovich, I., Englund, D. \& Toth, M. Solid-state single-photon emitters Nat. Photonics 10, 631 (2016).

16. Koperski, M. et al. Single photon emitters in exfoliated WSe2 structures. Nat. Nanotechnol. 10, 503-506 (2015).

17. Srivastava, A. et al. Optically active quantum dots in monolayer WSe2. Nat. Nanotechnol. 10, 491-496 (2015).

18. Chakraborty, C., Kinnischtzke, L., Goodfellow, K. M., Beams, R. \& Vamivakas, A. N. Voltage-controlled quantum light from an atomically thin semiconductor. Nat. Nanotechnol. 10, 507-511 (2015).

19. He, Y.-M. et al. Single quantum emitters in monolayer semiconductors. Nat. Nanotechnol. 10, 497-502 (2015).

20. Tonndorf, P. et al. Single-photon emission from localized excitons in an atomically thin semiconductor. Optica 2, 347-352 (2015).
21. Li, X. et al. Nonmagnetic quantum emitters in boron nitride with ultranarrow and sideband-free emission spectra. ACS Nano 11, 6652-6660 (2017).

22. Martínez, L. J. et al. Efficient single photon emission from a high-purity hexagonal boron nitride crystal. Phys. Rev. B 94, 121405 (2016).

23. Tran, T. T., Bray, K., Ford, M. J., Toth, M. \& Aharonovich, I. Quantum emission from hexagonal boron nitride monolayers. Nat. Nanotechnol. 11, 37 (2016).

24. He, X. et al. Carbon nanotubes as emerging quantum-light sources. Nat. Mater. 17, 663-670 (2018).

25. Pfab, R. J. et al. Aligned terrylene molecules in a spin-coated ultrathin crystalline film of p -terphenyl. Chem. Phys. Lett. 387, 490-495 (2004).

26. Toninelli, C., Early, K., Bremi, J. \& Renn, A. Near-infrared single-photons from aligned molecules in ultrathin crystalline films at room temperature. Opt. Express 18, 23986-23991 (2010).

27. Bernard, J., Fleury, L., Talon, H. \& Orrit, M. Photon bunching in the fluorescence from single molecules: a probe for intersystem crossing. J. Chem. Phys. 98, 850-859 (1993).

28. Li, Y., Shu, H., Wang, S. \& Wang, J. Electronic and optical properties of graphene quantum dots: the role of many-body effects. J. Phys. Chem. C. 119, 4983-4989 (2015).

29. Schumacher, S. Photophysics of graphene quantum dots: Insights from electronic structure calculations. Phys. Rev. B 83, 081417 (2011).

30. Wehmeier, M., Wagner, M. \& Müllen, K. Novel perylene chromophores obtained by a facile oxidative cyclodehydrogenation route. Chem. Eur. J. 7, 2197-2205 (2001)

\section{Acknowledgements}

The authors are grateful to D. Beljonne, J.-F. Roch, and V. Jacques for discussions. The authors thank C. Allain for her help on the PLE experiments. This work was partly funded by the JST-ANR program TMOL "Molecular Technology" project MECANO (ANR-14-JTIC-0002-01) and the ANR projet MAGMA (ANR-16-CE29-0027-01) and by a public grant overseen by the French National Research Agency (ANR) as part of the "Investissements d'Avenir" program (Labex NanoSaclay, reference: ANR-10-LABX0035), and by the GDR-I GNT. J.S.L is partially funded by "Institut Universitaire de France".

\section{Author contributions}

S.Z. and L.R. have performed the optical experiments. J.L. and L.O-C. have performed the synthesis of GQDs. S.C. have supervised the synthesis. L.R. and J.S.L. have supervised the optical experiments and the analysis of the data. All the authors (S.Z., J.L., L.R., L.OC., C.D., P.R., Y.C., C.V., K.M., A.N., S.C., and J.S.L.) have discussed on the results and participated to the writing of the paper.

\section{Additional information}

Supplementary Information accompanies this paper at https://doi.org/10.1038/s41467018-05888-w.

Competing interests: The authors declare no competing interests.

Reprints and permission information is available online at http://npg.nature.com/ reprintsandpermissions/

Publisher's note: Springer Nature remains neutral with regard to jurisdictional claims in published maps and institutional affiliations.

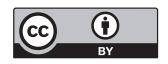

Open Access This article is licensed under a Creative Commons Attribution 4.0 International License, which permits use, sharing, adaptation, distribution and reproduction in any medium or format, as long as you give appropriate credit to the original author(s) and the source, provide a link to the Creative Commons license, and indicate if changes were made. The images or other third party material in this article are included in the article's Creative Commons license, unless indicated otherwise in a credit line to the material. If material is not included in the article's Creative Commons license and your intended use is not permitted by statutory regulation or exceeds the permitted use, you will need to obtain permission directly from the copyright holder. To view a copy of this license, visit http://creativecommons.org/ licenses/by/4.0/.

(C) The Author(s) 2018 